\title{
Tuberculosis contact investigation in an intermediate burden setting: implications from a large tuberculosis contact cohort in Taiwan
}

To the Editor:

There are still several unanswered research questions regarding tuberculosis (TB) contact investigations [1-4]. For example, up-to-date epidemiological studies regarding TB contacts in the area of intermediate incidence are not readily available [5]. The long-term risk of $\mathrm{TB}$, especially beyond 5 years, remains undetermined [6]. Risk factors for TB development among contacts are also not well defined [2]. To expand current understanding and knowledge of TB contact investigation, we recruited a population-based TB contacts cohort utilising the National Health Insurance Research Database of Taiwan, which included medical care claims data of $99 \%$ Taiwan residents [7].

Taiwan is a country with an intermediate TB burden. The incidence rate of TB was 72.5 per 100000 population in 2005 and decreased gradually to 45.7 in 2015 [8]. Supported by the national budget and a single, government-sponsored insurance medical service system, the completion rate of TB contact investigation has reached more than 95\% [9]. Participants were included if they had an outpatient diagnosis of International Classification of Diseases, ninth revision, clinical modification (ICD-9-CM) code of V01.1 or 795.5. At the same time, the participants should also fulfil the criteria of co-payment exemption. Under the National TB Program of Taiwan, TB contacts were exempt from a certain fraction of the actual cost of using the medical services, the so-called co-payment exemption, since July 2007. The date of this outpatient visit was set to be the cohort entry date. Contacts were followed until December 31, 2013. The primary outcome was development of active TB.

Early adherent isoniazid preventive therapy (IPT) was defined as starting isoniazid within 180 days after the entry date and lasting for at least 180 days. Non-adherent IPT was defined as starting isoniazid within 180 days of the entry date but lasting for fewer than 180 days. Contacts with ICD-9 code 795.5 or a prescription for IPT within 6 months after the entry date were defined as having latent tuberculosis infection (LTBI)

The Cox proportional hazard regression model was used to analyse factors associated with development of TB. Adjusted covariates included age, gender, medical visits within 3 months prior to the cohort entry date, contact area TB incidence, urban contact area, economic status, underlying diseases and steroid usage.

A total of 312322 participants were recruited in the cohort (figure 1). The median follow-up time was 955 days (IQR 468-1476 days). Among the 312322 TB close contacts, $0.7 \%$ developed active TB during follow-up ( $\mathrm{n}=2322)$. Among all TB close contacts, 140849 (45.1\%) were male and the median age was 36 years (IQR 21-52 years). The most common comorbidities were diabetes mellitus (5.8\%), chronic obstructive pulmonary disease $(2.1 \%)$, malignancy $(1.6 \%)$ and end-stage renal disease (1.2\%). Of the overall contacts, $88.6 \%$ had no previous underlying diseases and were considered to be healthy.

A total of 32474 contacts with LTBI were included. Among them, 1346 (4.1\%) developed active TB during follow-up; of these, $98.4 \%(\mathrm{n}=1324)$ developed LTBI within 1 year of contact. The median age of contacts with LTBI was 12 years old (IQR 7-25 years). The vast majority of LTBI contacts had no comorbidities

@ERSpublications

Taiwan TB contact cohort: $0.5 \% 1$-year yield, age $\geqslant 65$ as significant risk factor and elevated TB risk at 5th year http://ow.ly/9ZGr30dpkMc

Cite this article as: Lee M-R, Ho C-M, Lee C-H, et al. Tuberculosis contact investigation in an intermediate burden setting: implications from a large tuberculosis contact cohort in Taiwan. Eur Respir J 2017; 50: 1700851 [https://doi.org/10.1183/13993003.00851-2017]. 
FIGURE 1 Flowchart of patient recruitment. NHIRD: National Health Insurance Research Database; TB: tuberculosis; ICD-9. $\mathrm{CM}$ : International Classification of Diseases, 9th Edn, clinical modification; LTBI: latent tuberculosis infection.

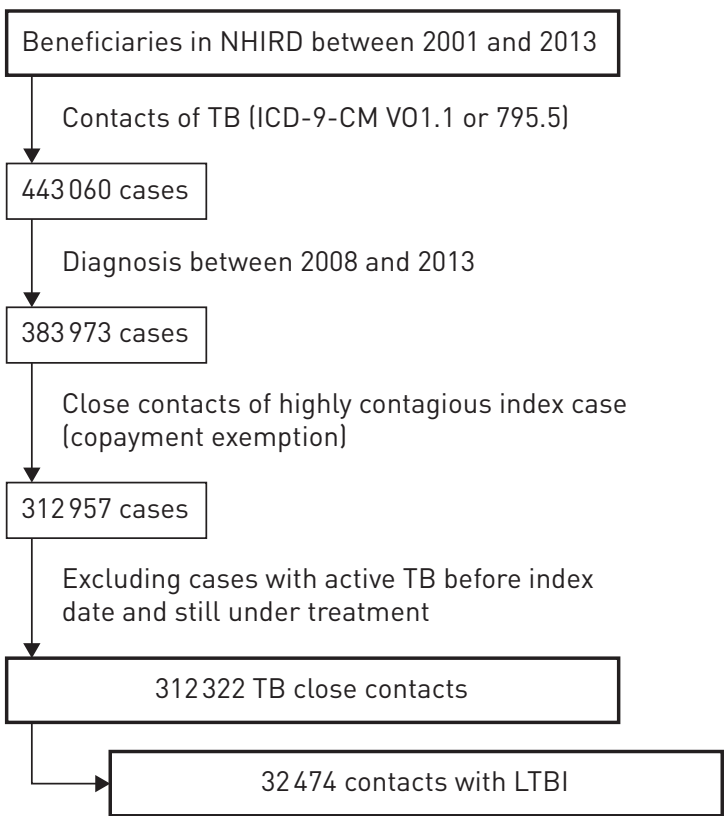

(95\%). $41 \%$ of all LTBI contacts received early adherent IPT with $0.2 \%$ (25/13435) of them developing active TB during follow-up, while $31 \%$ received non-adherent IPT with $0.7 \%(73 / 10032)$ developing active TB.

Among overall TB close contacts, the incidence of active TB was 3236, 889 and 415 (per 100000 person-years) in the first, second and third months, respectively. At 1 year, the incidence decreased to 202 (per 100000 person-years), and at the third year the rate decreased to 119 (per 100000 person-years). The incidence rate remained stable within the third to fifth year (143 per 100000 person-years) (figure 2), but always higher than the baseline population in Taiwan (the incidence rate of TB was 33.6 per 100000 person-years for those aged between 45 and 55 in 2015 in Taiwan) [8]. The highest TB incidence group was among those aged more than 65 years (634 at first year and 403 at the fifth year per 100000

FIGURE 2 Incidence of tuberculosis (TB) among contacts of major entities loverall, diabetes mellitus (DM), healthy, with comorbidity) and different age groups with time.

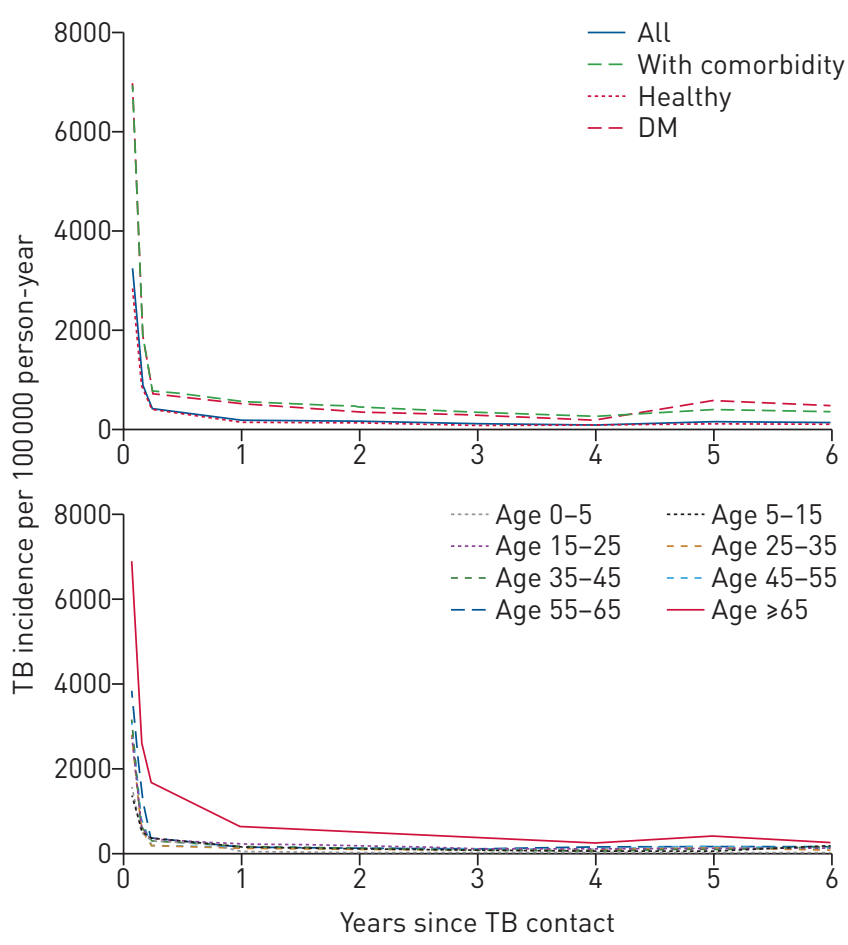


person-years) (figure 2). The cumulative percentage of active TB for all contacts was $35.4 \%, 45.0 \%$ and $49.4 \%$ at the first, second and third months after contact, respectively.

Among all TB contacts, major risk factors associated with active TB development in the Cox regression model included age (with age less than 5 years as the reference group, the risk was highest in contacts aged $\geqslant 65$ (HR 26.10, 95\% CI 17.37-39.23), and aged between 55 and 65 (HR 12.50, 95\% CI 8.26-18.91)), LTBI (HR 65.45, 95\% CI 60.02-71.37) and AIDS (HR 4.79, 95\% CI 2.14-10.71).

Among contacts with LTBI, the major risk factors associated with TB development included age (with age less than 5 years as the reference group, the risk was highest in contacts aged $\geqslant 65$ (HR 22.19, 95\% CI 14.4-34.19)), AIDS (HR 7.10, 95\% CI 2.62-19.29) and high contact area incidence (HR 2.94, 95\% CI $1.45-6.00)$.

Among contacts with LTBI, early adherent IPT was associated with a lower risk of active TB development (HR 0.02, 95\% CI 0.02-0.03) while non-adherent IPT remained associated with a lower risk of active TB development (HR 0.11, 95\% CI 0.09-0.14).

The long-term risk of TB among close contacts remained undetermined since most studies conducted previously were limited by the case number and follow-up period (usually less than 5 years) [3, 6]. Our study revealed that the TB risk was still higher than the general population 5 years after TB contact. While it can be impractical in public health policy to regularly follow such a large number of contacts for years, integrating contact history (even beyond years) as a risk factor for tuberculosis into routine clinical practice may be considered.

In a meta-analysis pooling five randomised control trials, those who received IPT had a relative risk of 0.31 of developing active TB [10]. Our study revealed a stronger association between IPT and TB risk reduction. This interpretation should be taken with caution because of the observational nature of our study. For example, after contacts were coded as LTBI because of positive tuberculin skin tests and referred, IPT would not be given for those suspected to have coprevalent TB.

In addition to what was already included in the guidelines, we found that older age, especially those above 65 years old, was associated with a significant risk for TB (HR 26.1, 95\% CI 17.37-39.23). The fact that older age was associated with significantly higher risk does not equal the necessity of treatment of LTBI among elderly people. The risk of TB drug-related hepatotoxicity and systemic drug reaction remains a concern, especially in the elderly population [11, 12]. If elderly contacts had some underlying comorbidities such as diabetes mellitus, however, the risk may be further elevated [13, 14]. Careful selection of patients is mandatory before initiating LTBI treatment among elderly people.

Our study also has limitations. We did not have data on an index case, especially sputum mycobacterial study results. Second, not until 2016 was universal screening for LTBI performed in Taiwan TB contact investigations. Also, the identification of LTBI is through a diagnosis code or use of IPT and has not yet been validated.

In conclusion, we found that under a setting of intermediate $\mathrm{TB}$ incidence, $\mathrm{TB}$ contact investigation resulted in $0.5 \%$ yield with a 1 -year follow-up. TB risk among contacts remained higher than the general population after a 5-year interval. Specific subgroups of contacts (e.g. age $\geqslant 65$, AIDS) were at higher risk for developing TB and warrant special attention.

Meng-Rui Lee $e^{1,2,3}$, Cheng-Maw $\mathrm{Ho}^{4}$, Chih-Hsin Lee ${ }^{5,6}$, Ming-Chia Lee ${ }^{7,8}$, Lih-Yu Chang ${ }^{1,2}$, Kai-Lun Yu ${ }^{1,2}$, Jen-Chung Ko ${ }^{1}$, Jann-Yuan Wang ${ }^{2}$, Jann-Tay Wang ${ }^{2}$ and Li-Na Lee, ${ }^{2,9}$

${ }^{1}$ Dept of Internal Medicine, National Taiwan University Hospital, Hsin-Chu Branch, Hsin-Chu, Taiwan. ${ }^{2}$ Dept of Internal Medicine, National Taiwan University Hospital, Taipei, Taiwan. ${ }^{3}$ Institute of Epidemiology and Preventive Medicine, College of Public Health, National Taiwan University, Taipei, Taiwan. ${ }^{4}$ Dept of Surgery, National Taiwan University Hospital, Taipei, Taiwan. ${ }^{5}$ Division of Pulmonary Medicine, Dept of Internal Medicine, Wan Fang Hospital, Taipei Medical University, Taipei, Taiwan. ${ }^{6}$ Division of Pulmonary Medicine, Dept of Internal Medicine, School of Medicine, College of Medicine, Taipei Medical University, Taipei, Taiwan. ${ }^{7}$ Dept of Pharmacy, New Taipei City Hospital, New Taipei, Taiwan. ${ }^{8}$ School of Pharmacy, College of Pharmacy, Taipei Medical University, Taipei, Taiwan. ${ }^{9}$ Dept of Laboratory Medicine, National Taiwan University Hospital, Taipei, Taiwan.

Correspondence: Jann-Yuan Wang, Dept of Internal Medicine, National Taiwan University Hospital, \#7, Chung-Shan South Rd, Zhongzheng Dist., Taipei 10002, Taiwan. E-mail: jywang@ntu.edu.tw

Received: March 182017 | Accepted after revision: May 292017

Support statement: This study was funded by the Taiwan Ministry of Science and Technology (NSC101-3114-Y-002-003, MOST104-2321-B-002-058) and the Ministry of Health and Welfare (MOHW105CDC-C-114-00103). The funders had no role in the study design, data analysis, and manuscript writing. Funding information for this article has been deposited with the Crossref Funder Registry. 
Conflict of interest: None declared.

Acknowledgement: We acknowledge Lin Hsien-Ho for providing opinions and suggestions on the design and analysis of this epidemiological study.

\section{References}

1 Erkens CG, Slump E, Verhagen M, et al. Monitoring latent tuberculosis infection diagnosis and management in the Netherlands. Eur Respir J 2016; 47: 1492-1501.

2 Erkens CG, Kamphorst M, Abubakar I, et al. Tuberculosis contact investigation in low prevalence countries: a European consensus. Eur Respir J 2010; 36: 925-949.

3 Sloot R, Schim van der Loeff MF, Kouw PM, et al. Risk of tuberculosis after recent exposure. A 10-year follow-up study of contacts in Amsterdam. Am J Respir Crit Care Med 2014; 190: 1044-1052.

4 Fox GJ, Barry SE, Britton WJ, et al. Contact investigation for tuberculosis: a systematic review and meta-analysis. Eur Respir J 2013; 41: 140-156.

5 Kim YJ, Chi YH, Lee JY, et al. In-hospital contact investigation among health care workers after exposure to pulmonary tuberculosis in an intermediate tuberculosis prevalence area: A prospective study. Arch Environ Occup Health 2016: 1-7.

6 Feng JY, Chen SC, Lee MC, et al. Is 1-year follow-up adequate for adult tuberculosis contacts? Eur Respir J 2015, 45: 1501-1504.

7 Wang JY, Sun HY, Wang JT, et al. Nine- to twelve-month anti-tuberculosis treatment is associated with a lower recurrence rate than 6-9-month treatment in human immunodeficiency virus-infected patients: a retrospective population-based cohort study in Taiwan. PLoS One 2015; 10: e0144136.

8 Centers for Disease Control. Statistics of Communicable Diseases and Surveillance Report and Prevention, Taiwan 2016. Atlanta, GA, CDC, 2016.

9 Ling DL, Liaw YP, Lee CY, et al. Contact investigation for tuberculosis in Taiwan contacts aged under 20 years in 2005. Int J Tuberc Lung Dis 2011; 15: 50-55.

10 Kahwati LC, Feltner C, Halpern M, et al. Screening for Latent Tuberculosis Infection in Adults: An Evidence Review for the US Preventive Services Task Force. Rockville, US Preventive Services Task Force, 2016.

11 Shu CC, Lee CH, Lee MC, et al. Hepatotoxicity due to first-line anti-tuberculosis drugs: a five-year experience in a Taiwan medical centre. Int J Tuberc Lung Dis 2013; 17: 934-939.

12 Sterling TR, Moro RN, Borisov AS, et al. Flu-like and other systemic drug reactions among persons receiving weekly rifapentine plus isoniazid or daily isoniazid for treatment of latent tuberculosis infection in the PREVENT tuberculosis study. Clin Infect Dis 2015; 61: 527-535.

13 Lee MR, Huang YP, Kuo YT, et al. Diabetes mellitus and latent tuberculosis infection: a systemic review and meta-analysis. Clin Infect Dis 2017; 64: 719-727.

14 Lee PH, Fu H, Lai TC, et al. Glycemic control and the risk of tuberculosis: a cohort study. PLoS Med 2016; 13: e1002072. 